\title{
Single-Pulse Measurement of Orbital Angular Momentum Generated by Microring Lasers
}

\author{
Robert C. Keitel, Boris le Feber, Krispin M. Dettlaff, Raphael Brechbühler, \\ Eva De Leo, Henar Rojo, and David J. Norris*
}

Optical Materials Engineering Lab, Dept. of Mechanical and Process Engineering, ETH Zurich, 8092 Zurich, Switzerland

$\begin{array}{lll}\text { Contents Topic } & \text { Page }\end{array}$

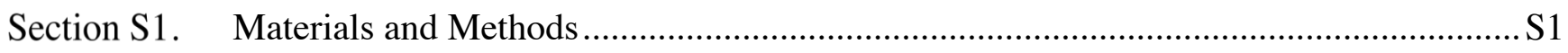

Section S2. Derivation of Fourier Images from OAM Lasers ..................................................... 2

Section S3. Effect of Adding an Aperture in the Fourier Plane ................................................... S12

Section S4. Rate-Equation Modelling of Lasing Dynamics …………........................................ 13

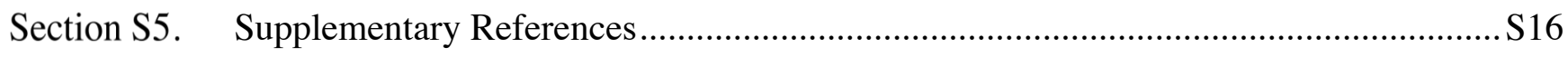

\section{Section S1. Materials and Methods}

Materials. 1-mm-thick, four-inch-diameter, single-side-polished, single-crystalline $\operatorname{Si}(100)$ wafers were purchased from Silicon Quest. Acetone (Technic France, Micropur VLSI Grade), isopropanol (IPA, Technic France, Micropur VLSI Grade), CSAR resist (AR-P 6200.04, Allresist GmbH), developer solution (AR 600-546, Allresist $\mathrm{GmbH}$ ), and buffered hydrofluoric acid (hydrogen fluoride/ammonium fluoride 1:7, Technic France, Micropurt VLSI Grade) used for template fabrication were provided by the Binnig and Rohrer Nanotechnology Center (BRNC) at IBM Zurich. Deionized (DI) water (18.2 M $\Omega$ ) was obtained from a MilliQ Advantage A10 water purification system. Octadecyltrichlorosilane (OTS, $96 \%$ ) was purchased from Merck KGaA. Bicyclohexyl (99\%, D79403), carbon tetrachloride $\left(\mathrm{CCl}_{4}, 99.5 \%, 289116\right)$, chloroform $(99.8 \%, 132950)$, and sulfuric acid $\left(\mathrm{H}_{2} \mathrm{SO}_{4}, 95-98 \%, 258105\right)$ were purchased from Sigma. Hydrogen peroxide $\left(\mathrm{H}_{2} \mathrm{O}_{2}, 30 \%\right.$, AnalaR Normapur) was purchased from VWR.

Dimensions of ring lasers. All ring lasers studied had a radius of $6 \mu \mathrm{m}$, and a width and height of $500 \mathrm{~nm}$. For circularly symmetric ring lasers, we used a rectangular grating at the inner sidewall extending $100 \mathrm{~nm}$ in the radial 


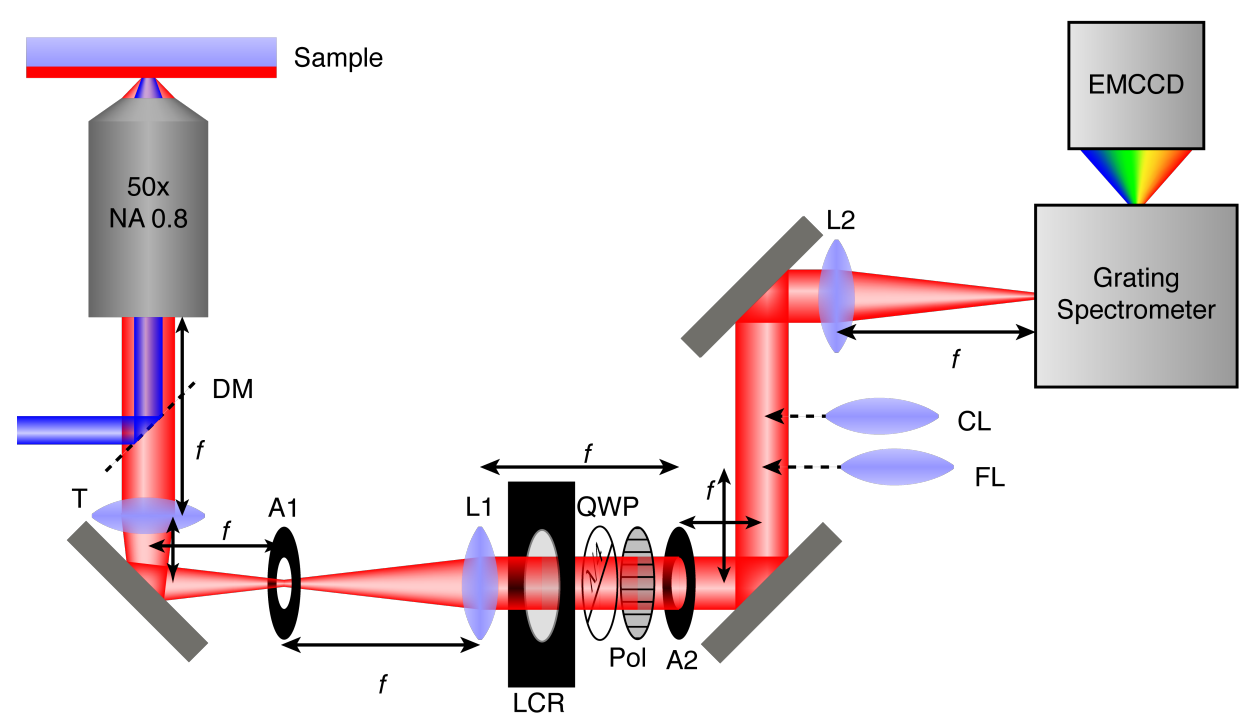

Figure S1. Sketch of the optical path, consisting of a pump laser (not shown), a dichroic mirror (DM), objective, tube lens (T) with focal length $200 \mathrm{~mm}$, apertures (A1 and A2), relay lenses (L1 and L2) with focal length $200 \mathrm{~mm}$, a liquid-crystal retarder (LCR), an optional quarter-wave plate (QWP) and polarizer (Pol), optional cylindrical $(\mathrm{CL})$ and Fourier lens (FL), spectrometer, and EMCCD camera.

and $200 \mathrm{~nm}$ in the azimuthal directions. For the blazed-grating design, the grating was also placed at the inner sidewall, but extended $120 \mathrm{~nm}$ in the radial direction at the thickest section. The lowest point of one grating element coincided with the highest point of the neighboring element. For unidirectional lasers, the grating elements were moved to the outer sidewall and had a size of $100 \mathrm{~nm}$ in the azimuthal and $50 \mathrm{~nm}$ in the radial directions. The S-shaped coupler had a width of $500 \mathrm{~nm}$ and was separated by $50 \mathrm{~nm}$ from the ring at the closest points.

\section{Section S2. Derivation of Fourier Images from OAM Lasers}

Fourier transform of the electric field. The experimentally observed Fourier images and polarizations can be explained with an analytical model. We start with the Fourier transform ( 7 ) of the local scattered field: ${ }^{\mathrm{S} 1 \mathrm{~S} 2}$

$$
\widehat{\boldsymbol{E}}\left(k_{x}, k_{y} ; z=0\right)=\boldsymbol{F}[\boldsymbol{E}(x, y, 0)] \propto \iint_{-\infty}^{\infty} \boldsymbol{E}(x, y, 0) \exp \left[-i\left(k_{x} x+k_{y} y\right)\right] \mathrm{d} x \mathrm{~d} y
$$

In our notation, bold variables denote vector quantities and the "hat" denotes a variable in Fourier space. The Fourier image is measured with a Fourier lens in the beam path. This lens transforms incident spherical waves into plane waves. As a result, while the Fourier image is a function of the in-plane wavevectors $k_{x}$ and $k_{y}$, the phase is evaluated on a sphere. Because the effective distance travelled by the field is independent of $\rho=\left(k_{x}+k_{y}\right)^{1 / 2}$, as long as the reference sphere is big compared the scattering structure, no additional phase factors appear. On the other 
hand, the far-field, which is often used to describe emission patterns, is evaluated on a plane. While this quantity gives similar insight, it is mathematically more complicated, because light arriving at the reference plane has to travel a distance dependent on the polar angle. Also, the differential area element on the reference plane depends on the polar angle. Thus, the Fourier image obtained by back-focal-plane imaging can be directly calculated as the square modulus of eq S1 for in-plane wavevectors that are within the numerical aperture of the objective used.

For geometries with periodic outscatterers, calculation of the emission pattern can be greatly simplified by decomposing the scattered field into the convolution of a periodic lattice, $\boldsymbol{g}(x, y, 0)$, and a unit cell, $\boldsymbol{u}(x, y, 0)$ :

$$
\boldsymbol{E}(x, y, 0)=\boldsymbol{g}(x, y, 0) \otimes \boldsymbol{u}(x, y, 0)
$$

When plugging this expression into eq S1 and using the convolution theorem, we obtain a disentangled version of the Fourier transform of the electric field:

$$
\widehat{\boldsymbol{E}}\left(k_{x}, k_{y} ; 0\right) \propto \mathcal{Z}[\boldsymbol{g}(x, y, 0)] \boldsymbol{z}[\boldsymbol{u}(x, y, 0)] .
$$

Due to the inverse relationship between length scales in real and in Fourier space, the unit-cell term corresponds to higher spatial frequencies. Thus, the angular position of emission maxima and minima is given by the Fourier transform of the periodic term. The unit cell provides an amplitude and phase envelope. For completeness, we mention that our treatment considers the outscatterers as point sources. For a more complete model, a spatial envelope function, which after Fourier transformation will give the linewidth and shape of the emission maxima, should be included.

Scalar theory for the Fourier images. We start by deriving the Fourier-transformed electric field generated by a counter-clockwise-propagating lasing mode in a scalar model. Due to the symmetry of ring lasers, the problem is best treated in polar coordinates. Despite the different properties of polar and Cartesian coordinates, the convolution theorem is still applicable. ${ }^{\mathrm{S} 3}$ The lattice can be reduced to an azimuthal series of periodic Dirac delta functions, one for each of the $q$ outscatterers. In the radial direction, it is given by a single Dirac delta function positioned at the inner radius of the ring, where the outscatterers are located. The angular position of the $m^{\text {th }}$ outscatterer is given by $2 \pi m / q$. Because they are all coherently driven by the travelling lasing mode, they radiate with a relative phase of $\exp \{i p 2 \pi m / q\}$, with $p$ as the azimuthal mode number. The periodic function is thus given by: 


$$
g(r, \psi) \propto \frac{1}{r} \delta\left(r-r_{\text {ring }}\right) \sum_{m=1}^{q} \delta(\psi-2 \pi m / q) \exp \{i p 2 \pi m / q\}
$$

where $r$ and $\psi$ are the radius and azimuthal angle in real-space, $q$ denotes the number of outscatterers, and $r_{\text {ring }}$ is the radius of the ring. The Fourier transform of this is: ${ }^{\mathrm{S} 3}$

$$
\mathcal{F}[g(r, \psi)]=\widehat{G}(r, \psi) \propto q \sum_{n=-\infty}^{\infty} i^{-(p-n q)} \exp [i(p-n q) \varphi] J_{p-n q}\left(\rho r_{\text {ring }}\right)
$$

where $\rho=\left(k_{x}+k_{y}\right)^{1 / 2}$ and $\varphi$ are the radial and azimuthal variables of the Fourier image, $n$ is the diffraction order, and $J_{\alpha}$ is the Bessel function of the first kind of order $\alpha$. While all terms in the sum in eq S5 are necessary to describe the full field, only the $n=1$ term has in-plane momentum low enough to radiate to free space for ring geometries where $p \approx q$. All other terms have appreciable intensity only for $\rho>2 \pi / \lambda$, which makes them evanescent. Accordingly, we expect to see the square modulus of Bessel functions of the order $p-q$ in the Fourier image, which is qualitatively consistent with our measurements (see Figure $2 \mathrm{a}$ in the main text). An intuitive way to rationalize the emission patterns is to consider the length scales in the azimuthal and radial direction. While in the azimuthal direction the spacing of grating elements is on the order of the wavelength (i.e., we see only one azimuthal diffraction order $n=1$ in the far-field), in the radial direction the microring is significantly bigger than the wavelength (i.e., several radial diffraction orders are visible as concentric rings in the Fourier image).

Vector theory for the Fourier images. An accurate description of the problem must include the vector nature of light. A Cartesian basis is not well-suited for the description, as a source that is displaced along the microring is not just translated but also rotated, which is cumbersome to express in a Cartesian basis. To circumvent this, we use a basis of right- and left circular unit vectors and an out-of-plane unit vector. We define circular terms, making use of the point-of-view-of-the-source convention and express time-dependencies with $\exp (-i \omega t)$. In this convention, right-circular polarization is described by the Jones vector $\boldsymbol{e}_{R}=\frac{1}{\sqrt{2}}\left(\begin{array}{l}1 \\ i\end{array}\right)$ and left-circular polarization by $\boldsymbol{e}_{L}=\frac{1}{\sqrt{2}}\left(\begin{array}{c}1 \\ -i\end{array}\right)$. However, a circular dipole located at the inside of the ring is defined using the radial and azimuthal unit vector. In a laboratory reference frame, using unit vectors $\boldsymbol{e}_{x}$ and $\boldsymbol{e}_{y}$, it will turn gradually when progressing along the ring. This rotation can be taken into account as a phase shift when working in a circular basis. For a right-circular unit vector located at azimuthal position $\psi$, we obtain: 


$$
\frac{1}{\sqrt{2}}\left(\boldsymbol{e}_{r}+i \boldsymbol{e}_{\psi}\right)=\frac{1}{\sqrt{2}}\left[\left(\begin{array}{c}
\cos (\psi) \\
\sin (\psi)
\end{array}\right)+i\left(\begin{array}{c}
-\sin (\psi) \\
\cos (\psi)
\end{array}\right)\right]=\frac{1}{\sqrt{2}}\left[\exp (-i \psi)\left(\begin{array}{l}
1 \\
i
\end{array}\right)\right]
$$

and for a left-circular unit vector:

$$
\frac{1}{\sqrt{2}}\left(\boldsymbol{e}_{r}-i \boldsymbol{e}_{\psi}\right)=\frac{1}{\sqrt{2}}\left[\left(\begin{array}{c}
\cos (\psi) \\
\sin (\psi)
\end{array}\right)-i\left(\begin{array}{c}
-\sin (\psi) \\
\cos (\psi)
\end{array}\right)\right]=\frac{1}{\sqrt{2}}\left[\exp (i \psi)\left(\begin{array}{c}
1 \\
-i
\end{array}\right)\right]
$$

We thus transform from the local coordinate system at the ring to circular terms defined in Cartesian labcoordinates by multiplying by a phase factor of $\exp (-i \psi)$ on the right-handed and $\exp (i \psi)$ on the left-handed unit vector. When only considering the first diffraction order $(n=1)$, eq S5 modifies for a vector model to:

$$
\begin{aligned}
& \widehat{\boldsymbol{G}}(\rho, \varphi) \propto q i^{-(p-q)}\left\{a J_{|p-q|}\left(\rho r_{\text {ring }}\right) \exp [i(p-q) \varphi] \hat{\boldsymbol{e}}_{z}\right. \\
& \left.+i b J_{p-q-1}\left(\rho r_{\text {ring }}\right) \hat{\boldsymbol{e}}_{R} \exp [i(p-q-1) \varphi]+i^{-1} c J_{p-q+1}\left(\rho r_{\text {ring }}\right) \hat{\boldsymbol{e}}_{L} \exp [i(p-q+1) \varphi]\right\} .
\end{aligned}
$$

Eq S8 shows that the lattice thus contributes OAM components with topological charge $p-q, p-q-1$, and $p-q+1$, with amplitudes scaled by Bessel functions of respective order. The variables $a, b$, and $c$ are the complex amplitudes of the different contributions. Therefore, for a given resonance, in principle, three different topological charges could be observed in the emission, corresponding to emission from out-of-plane, right-circular, and leftcircular dipoles. The vectors $\hat{\boldsymbol{e}}_{z}, \hat{\boldsymbol{e}}_{R}$, and $\hat{\boldsymbol{e}}_{L}$ denote the unit-cell emission pattern from the respective dipole term. Emission from the unit cell. To first approximation, we can treat the azimuthal grating as a chain of dipole emitters driven by the lasing mode. We construct circular dipoles by the superposition of $x$ - and $y$-oriented linear dipoles with a phase shift of $\pm \pi / 2$ for right- and left-circular dipoles, respectively. The emission patterns of each of the two linear dipoles can be calculated to be proportional to $\boldsymbol{r} \times \boldsymbol{p} \times \boldsymbol{r}$, with $\boldsymbol{p}$ denoting the orientation of the linear dipole and $\boldsymbol{r}$ the direction of emission. Such a circular dipole emits intensity proportional to:

$$
I_{\text {circdipole }}(\theta, \varphi) \propto[3+\cos (2 \theta)] / 4
$$

when expressed in spherical coordinates. As we collect intensity as a function of the in-plane wavevector in our experiment, we can transform this expression to:

$$
I_{\text {circdipole }}(\rho, \varphi) \propto\left(1-\rho^{2} / 2 k_{0}^{2}\right) /\left(1-\rho^{2} / k_{0}^{2}\right)^{1 / 2},
$$

where we used $\rho / k_{0}=\sin (\theta)$ with wavevector $k_{0}=2 \pi / \lambda$. The division by $\left(1-\rho^{2} / k_{0}^{2}\right)^{1 / 2}$ is needed to account for the change of the differential area element when performing the projective transformation from spherical to polar 


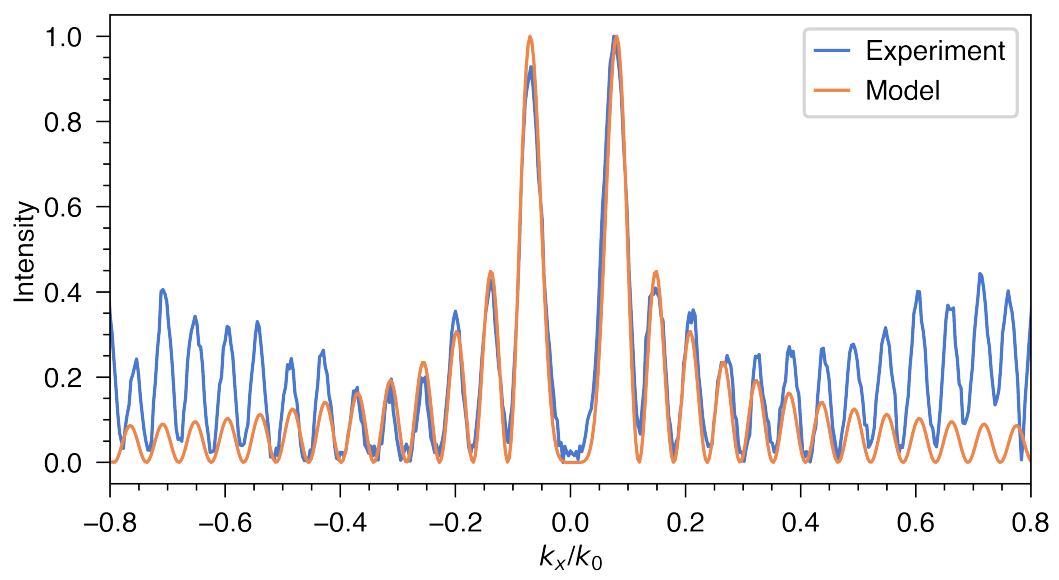

Figure S2. Comparison of experimentally measured and calculated Fourier images (square modulus of the rightcircular term of eq S8 with $r_{\text {ring }}=5.7 \mu \mathrm{m}, \lambda=640 \mathrm{~nm}, p-q-1=3$ multiplied by eq S10).

coordinates. ${ }^{\mathrm{S} 2} \mathrm{Eq} \mathrm{S10}$ can be multiplied by the square modulus of eq S8, considering only the right-circular term, to obtain the overall Fourier image of the light emitted from outscatterers described by right-circular dipoles.

The simplified model excellently captures the dependence of intensity on in-plane wavevector for emission angles up to approximately 25 degrees $\left(\rho / k_{0}=0.43\right)$, as shown in Figure S2. Beyond this, we experimentally observe an increase of intensity with increasing angle while the model predicts a drop. This could be caused by the extended size of the ring in the out-of-plane direction or reflection from the interfaces between quantum dots, epoxy, glass, and air, which are all not explicitly considered in the simple dipole model.

Figure $2 \mathrm{~b}$ in the main text shows a complicated polarization pattern. This pattern can be analyzed more quantitatively by recording the Fourier image under four different polarizer angles. Figure S3a shows the extracted angle and degree of linear polarization. The observed polarization pattern can be qualitatively explained by the circular-dipole model. The degree of linear polarization emitted by a circular dipole is given by:

$$
\left(\rho / k_{0}\right)^{2} /\left[2-\left(\rho / k_{0}\right)^{2}\right]
$$

and the angle of linear polarization by:

$$
\arg [-\exp (i 2 \varphi)] / 2
$$

where $\arg []$ evaluates the phase angle in radians. Eqs S11 and S12 are visualized in Figure S3b. While we observe perfectly circularly polarized light with a single OAM value of $p-q-1$ close to the surface normal of the sample, indicated by a degree of linear polarization of 0 , under higher angles, an additional term starts to contribute, which is left circularly polarized and carries OAM $p-q+1$. This additional OAM component under large angles leads to 

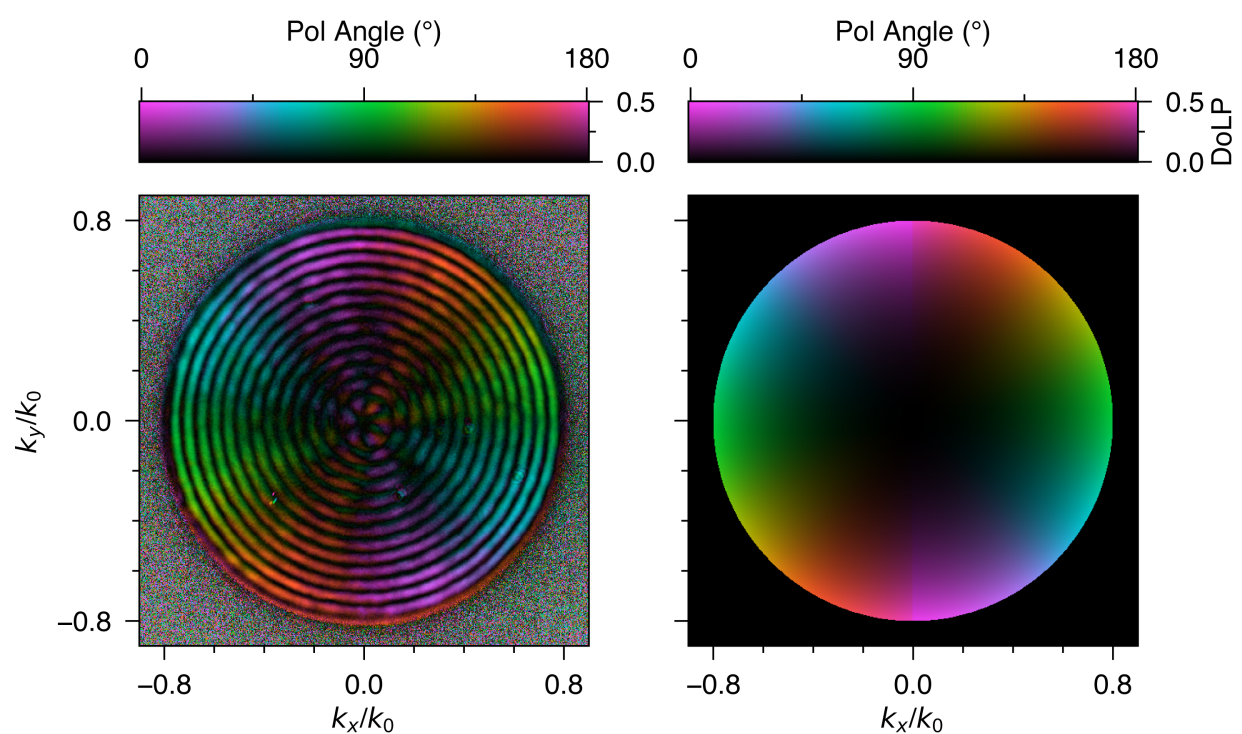

Figure S3. (a) Angle and degree of linear polarization (Pol Angle and DoLP, respectively) processed from Fourier images of the device discussed in Figure 2 of the main text under four different polarizer orientations. (b) Pol Angle and DoLP of a circular dipole emitter, calculated with eqs S11 and S12.

an increased degree of linear polarization. Consequently, the polarization under high angles becomes elliptical with the major axis aligned with the azimuth, as shown in Figure S3.

This finding provides a simple explanation for the increasingly azimuthal polarization of the emission of our rings under higher angles, because a circular dipole does not emit circularly polarized light under high polar angles. In contrast to previous work, we thus observe radiation from phased circular rather than linear-dipole scatterers. ${ }^{\mathrm{S}, \mathrm{S} 5} \mathrm{We}$ attribute this to lasing in the quasi-transverse-magnetic (TM, electric field in the plane of the ring), rather than the quasi-transverse-electric (TE, electric field perpendicular to the plane of the ring) mode. The coupling between the direction of propagation of a bound mode to a circular polarization state in the far-field is reminiscent of the spin-momentum locking observed in different systems where the spin of the emitted photon is linked to the transverse momentum of a surface mode ${ }^{\mathrm{S} 6, \mathrm{S7}}$ For a counter-clockwise-propagating mode and a grating at the inner sidewall, the right-circularly polarized component dominates ( $a$ and $c$ are small in eq S8), while for grating elements at the outer sidewall, the left-circularly polarized component of eq S8 becomes strongest ( $a$ and $b$ are small in eq S8). The observation of two pure OAM modes, corresponding to light scattered from the clockwise and counter-clockwise-propagating modes in Figure 3 in the main text, is made possible by an aperture in a Fourier plane that blocks higher angles (A2 in Figure S1). Under the experimentally considered angles, the 

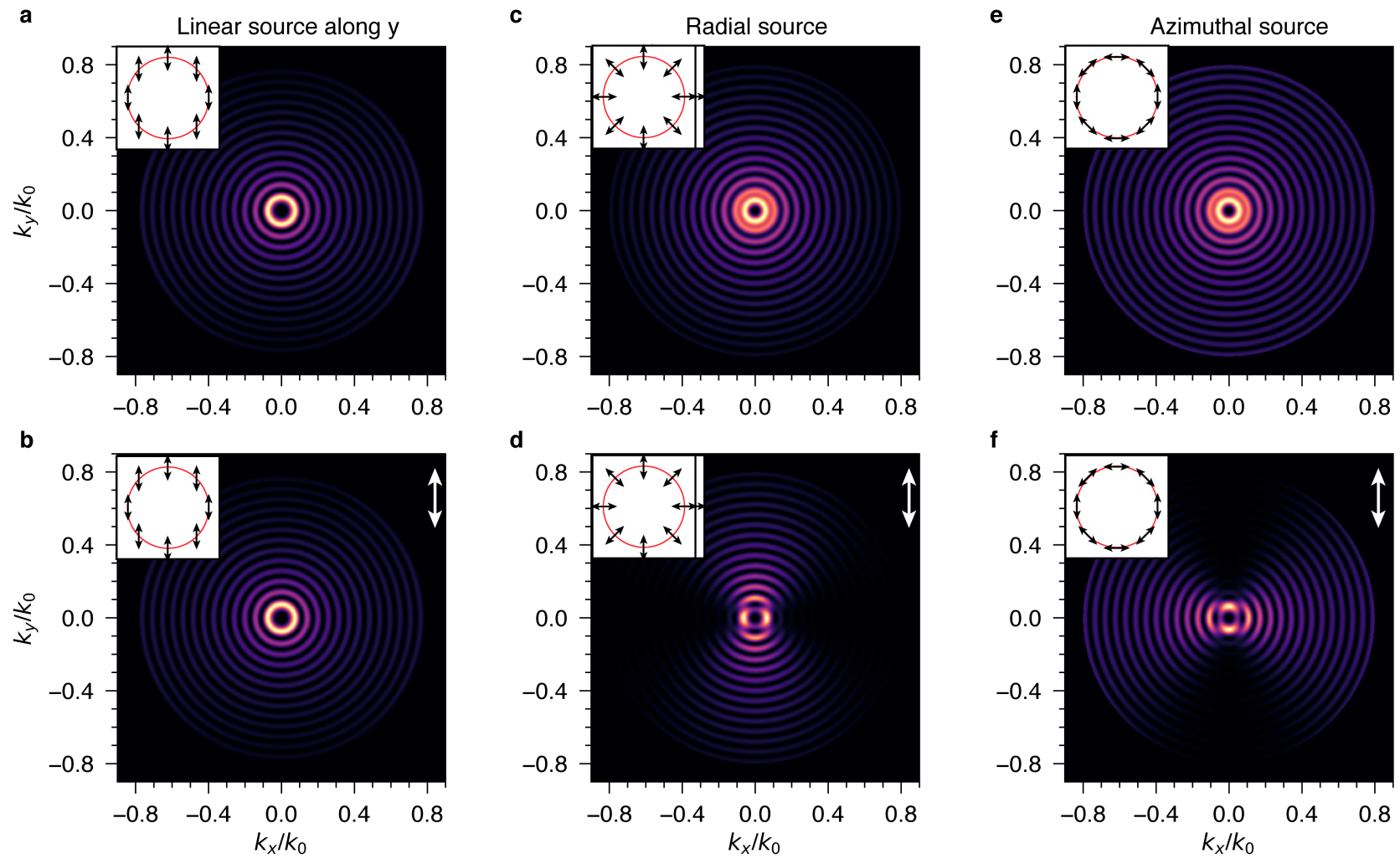

Figure S4. Calculated total Fourier image (top row) and filtered through a vertical polarizer (bottom row) for linear sources oriented along $y(a, b)$, linear sources oriented along the radial direction (c, d), and linear sources oriented along the azimuthal direction (e, f).

scatterers emit almost perfectly circularly polarized light, and thus single-valued OAM for each of the two counterpropagating lasing modes.

Polarization of Fourier images with different unit cells. In our current devices we find that the emission can be described by assuming that each grating element radiates like a circular point dipole. However, our model also allows us to straightforwardly calculate the Fourier image for other configurations. In Figure S4a, we show the Fourier image assuming that each grating element radiates like a linear dipole oriented along the $y$ axis. Because the source does not rotate when progressing along the ring, the equation $l=p-q$ holds in this case in contrast to all other cases. In the framework of our model, this is expressed by the first term in the bracket in eq S8. For this case, we observe a pure OAM output linearly polarized along y up to high angles (Figure S4b). While we observe a pure OAM output, comparable to our experimental results (Figures $2 \mathrm{~b}$ and $3 \mathrm{~b}$ ), the arrangement shown in Figure $\mathrm{S} 4 \mathrm{a}$ (inset) is not compatible with the rotational symmetry in our devices.

If instead each grating element radiates like a radially oriented dipole (Figure $\mathrm{S} 4 \mathrm{c}$, inset), we observe radiation in an OAM superposition state as explained by eq S8 with $b=1$ and $c=1$ (Figure S4c). This can readily be seen 
in the Fourier image where the innermost ring is not well defined but a double ring. The polarization under narrow angles is complicated and then turns to purely radial polarization under higher polar angles (Figure S4d). For an azimuthal source (eq S8 with $b=1$ and $c=-1$, Figure S4e) we observe a Fourier image similar to a radial source. The only differences are an increased intensity at high polar angles (Figure S4e) and a polarization pattern rotated by $90^{\circ}$ compared to the radial case (Figure $\mathrm{S} 4 \mathrm{f}$ ).

Fourier images from clockwise ring-laser modes. Fourier images from a clockwise ring-laser mode can be obtained using the same equation as above with modified signs. For a clockwise-propagating mode, eq S4 modifies to:

$$
g(r, \psi)=\delta\left(r-r_{\text {ring }}\right) \sum_{m=1}^{q} \delta(\psi-2 \pi m / q) \exp (-i p 2 \pi m / q)
$$

As a result, eq S5 modifies to:

$$
\mathcal{F}[g(r, \psi)]=\widehat{G}(r, \psi)=2 \pi \sum_{n=-\infty}^{\infty} i^{-(-p-n q)} \exp [i(-p-n q) \varphi] J_{-p-n q}\left(\rho r_{\text {ring }}\right)
$$

which means that for clockwise-propagating modes, only the $n=-1$ diffraction order has low enough OAM to propagate into the far field. Eq S8 modifies to:

$$
\begin{aligned}
& \widehat{\boldsymbol{G}}(\rho, \varphi)=2 \pi i^{-(p-q)}\left\{a J_{-p+q}\left(\rho r_{\text {ring }}\right) \hat{\boldsymbol{e}}_{z} \exp [i(-p+q) \varphi]\right. \\
& \left.+i b J_{-p+q-1}\left(\rho r_{\text {ring }}\right) \hat{\boldsymbol{e}}_{R} \exp [i(-p+q-1) \varphi]+i^{-1} c J_{-p+q+1}\left(\rho r_{\text {ring }}\right) \hat{\boldsymbol{e}}_{L} \exp [i(-p+q+1) \varphi]\right\},
\end{aligned}
$$

and we observe predominantly the left-circularly polarized component if the grating elements are placed at the inner sidewall.

Effect of pulse-to-pulse fluctuations on Fourier images. The polarimetry shown in Figure 2 in the main text is averaged over many shots. In light of the strong pulse-to-pulse fluctuations that we observe later in the manuscript, it remains to be shown that such a measurement can provide insight into the emission characteristics of the laser. In Figure S5 we present calculated Fourier images viewed through a linear polarizer. The calculation for a collection of right-circular dipole sources driven by a counter-clockwise-propagating lasing mode (Figure S5a) shows strong similarity to our experimental observation. However, we know from later measurements that during most pulses clockwise and counter-clockwise lasing coexists. In this case, the Fourier image viewed through a linear polarizer looks drastically different. We observe $2 l$ intensity minima (Figure S5b). However, the angle under 

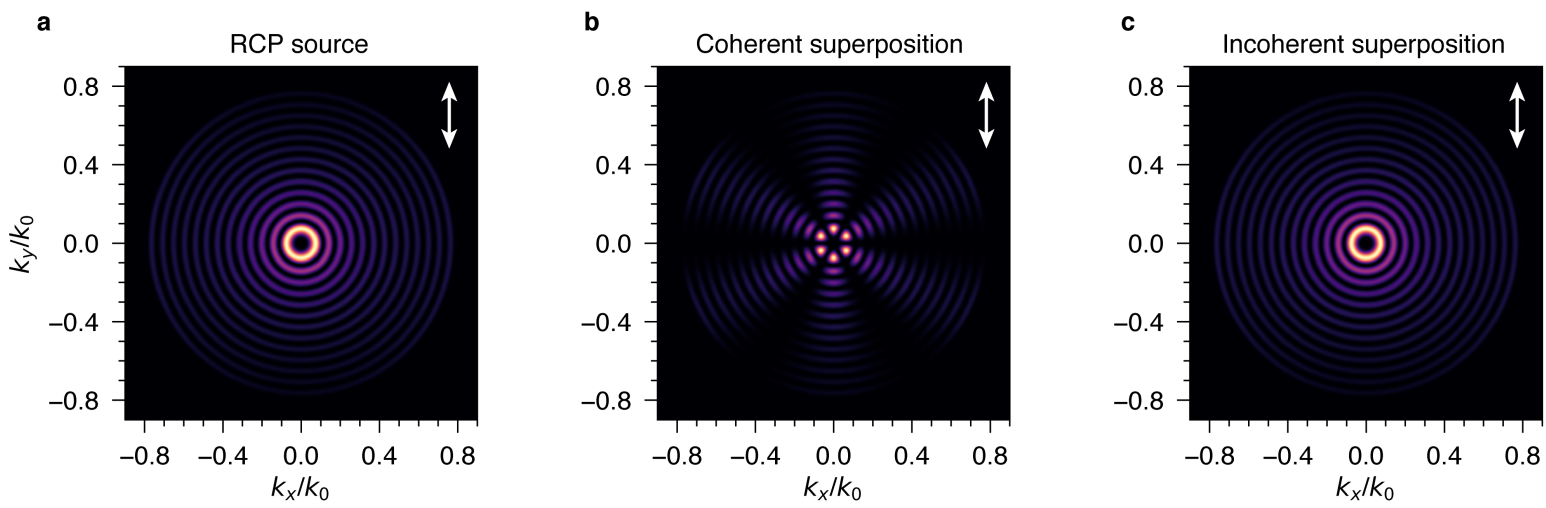

Figure S5. Calculated Fourier images viewed through a linear polarizer. (a) For a purely right-circular dipole originating from a counter-clockwise-propagating lasing mode. (b) A coherent superposition of right- and leftcircular dipoles originating from a combination of clockwise and counter-clockwise lasing modes shows $2 l$ intensity minima. (c) Averaging over different relative phases between the clockwise and counter-clockwise modes washes out the minima seen in (b). The linear polarization properties of an incoherent sum are equal to those of the pure circular dipole.

which the minima appear depends on the relative phase between the clockwise and counter-clockwise modes. If we average over different phases, which are random in the absence of significant scattering, we obtain a Fourier image which is identical to the one for the pure counter-clockwise mode (Figure S5c). Accordingly, when averaging over many shots, the linear polarization properties of an incoherent sum of clockwise and counterclockwise lasing modes are identical to the one of a pure clockwise mode. The only difference is that a single mode emits circularly polarized light under narrow angles; this circular polarization is averaged out for the incoherent mixture. For this reason, probing circular polarization does not provide further insight in this case.

Coherent coupling of counter-propagating modes. Revisiting the Fourier image shown in Figure 2a in the main text, one feature has not yet been accounted for by the above analytical treatment. Namely, we observe a weak high-frequency polarization modulation in the innermost concentric ring in the image when a linear polarizer is placed in the beam path. In the previous paragraph, we showed that the interference effects between emission from clockwise and counter-clockwise lasing do not contribute on average if their relative phase is random (i.e. they are incoherent). Scattering can lead to a preferred relative phase, giving the two modes a degree of coherence. We model this by considering the emission from a strong counter-clockwise mode (emitting right-circular light with OAM $l$ ) interfering with a weak clockwise mode (emitting left-circular light with OAM $-l$ ). The resulting electric field in the Fourier image for narrow angles is described along the azimuthal direction by: 

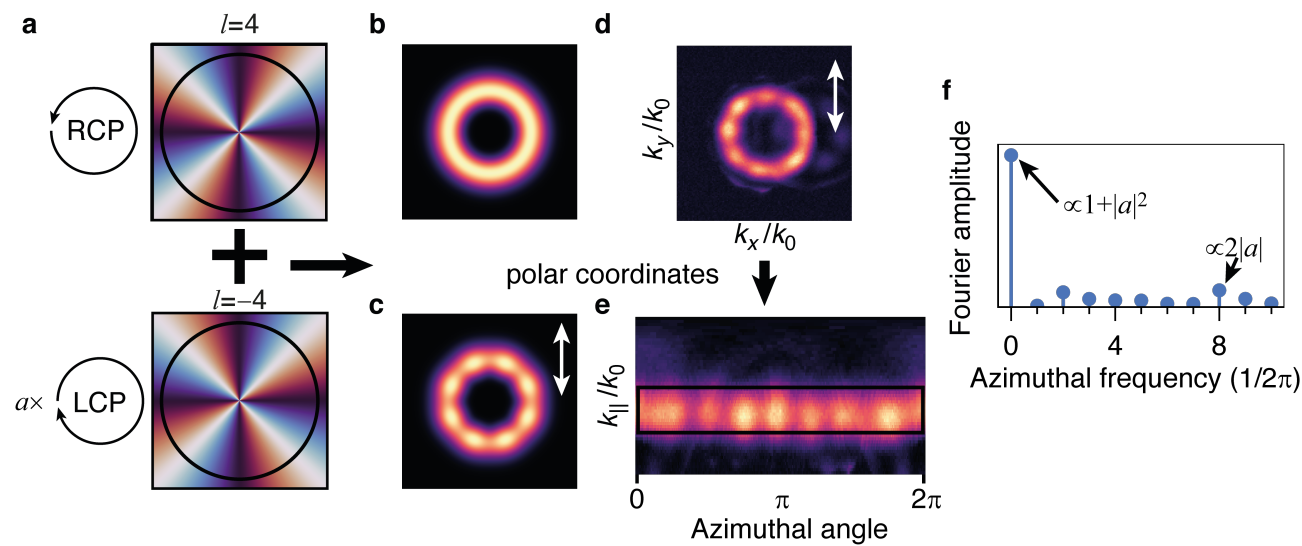

Figure S6. Interference of (a) right-circularly polarized light with $l=4$ and left-circularly polarized light with $l=-4$ and relative complex amplitude $a$ shows (b) no azimuthal intensity modulation. (c) When viewed through a linear polarizer (aligned as shown with the double arrow), an intensity modulation becomes apparent. (d) We observe the same effect in our experimental Fourier images. Note that these data were acquired with a diffraction grating in the optical path to horizontally displace other lasing modes (visible at the right of the image), and we used an iris in a Fourier plane to focus on the narrow emission angles. (e) Same data, but transformed into polar coordinates. (f) Fourier transform along the azimuthal angle, as indicated by the box in (e) allows direct quantification of the modulation depth (azimuthal frequency $=8$ ) and the offset (azimuthal frequency $=0$ ).

$$
2^{-1 / 2}\left(\begin{array}{l}
1 \\
i
\end{array}\right) \exp (i l \varphi)+a \cdot 2^{-1 / 2}\left(\begin{array}{l}
1 \\
i
\end{array}\right) \exp (i l \varphi)=2^{-1 / 2}\left[\begin{array}{c}
\exp (i l \varphi)+a \exp (-i l \varphi) \\
i(\exp (i l \varphi)+a \exp (-i l \varphi))
\end{array}\right],
$$

where $a=|a| \exp \left\{i \varphi_{a}\right\}$ is a complex number (different from the $a$ in eqs S8 and S15), expressing the relative amplitude and phase of the coherent counter-propagating mode (see Figure S6a). Because both polarizations are orthogonal to each other, the intensity of eq S16 is constant and proportional to $1+|a|^{2}$ (compare Figure S6b). However, the interference of the two fields causes a modulation of the polarization, which can be seen when adding a linear polarizer to the optical path. If we evaluate the intensity of the vertically polarized component, we find:

$$
\begin{aligned}
& 1 / 2\left\{[\exp (i l \varphi)-a \exp (-i l \varphi)]\left[\exp (-i l \varphi)-a^{*} \exp (i l \varphi)\right]\right\} \\
& =1 / 2\left(1+|a|^{2}\right)-|a| \cos \left(2 l \varphi-\varphi_{a}\right)
\end{aligned}
$$

This means that coherent coupling between the counter-propagating modes causes an azimuthal modulation of frequency $2 l$ in the vertically polarized intensity, as shown in Figure S6c.

We stress that eq S17 holds for each individual laser pulse. However, in the absence of a coupling mechanism, the relative phase between the two modes, given by the phase of $a$, takes a random value for each pulse, and the interference pattern observable through a linear polarizer would wash out, as shown in Figure S5c. Thus, the 
observation of the high-frequency modulation, when viewing the Fourier image through a linear polarizer and averaging over many laser pulses, indicates coherent coupling of the counter-propagating modes. Such a coupling can be caused by any breaking of the rotational symmetry, for example slightly defective grating elements or other point defects.

At first glance, this coupling seems contradictory to our single-shot results, indicating strong mode competition (Figures 4 and 5 of the main text). However, the degree of coupling can be extracted from the modulation depth of the observed Fourier image (Figure S6d). The measured Fourier image is transformed into a polar coordinate system around the center of the mode of interest (Figure S6e). A Fourier decomposition over the azimuthal variable around the radius of maximum intensity allows us to quantify the modulation depth, by comparing the amplitude of the peak at the frequency of interest with the peak at 0 frequency in the Fourier spectrum (Figure S6f). We find that the peak at $2 l$ has a height of around $10 \%$ of the 0 -frequency peak. Together with eq S17, this indicates that the average coherent counter-propagating mode has a field amplitude $a$ of $5 \%$ relative to the main mode. As the power in the mode is proportional to the square of the field amplitude, this means that the relative power of the coherent counter-propagating mode is just $0.25 \%$ of the main mode. Accordingly, coherent coupling is small in our devices, in good agreement with our single-pulse statistics.

\section{Section S3. Effect of Adding an Aperture in the Fourier Plane}

To obtain images from which the OAM can easily be obtained from our ring lasers, the use of an aperture (A2 in Figure S1) to block emission beyond the innermost ring in the Fourier image is crucial. We now demonstrate the importance of A2. Due to the finite size of the source, the Fourier image does not only show an azimuthal phase variation, but also a phase jump in the radial direction at each intensity minimum (shown in Figure S7a). Because the electric field in the Fourier plane is given by multiplying eq S8 with the square root of eq S10, we can simulate the effect of the cylindrical lens by taking the 1D Fourier transform along the $k_{x}$ axis. The resulting pattern is shown in Figure S7b. While the OAM can be determined by counting the minima between the two intensity maxima (in this case four), interference effects due to the phase jumps in the radial direction give rise to several local intensity minima that obscure the pattern. The effect of the radial phase jumps can be removed by closing aperture A2 to only transmit the innermost ring of the Fourier image (shown in Figure S7c). The resulting calculated image after focusing with the cylindrical lens is shown in Figure S7d. This calculated image closely 

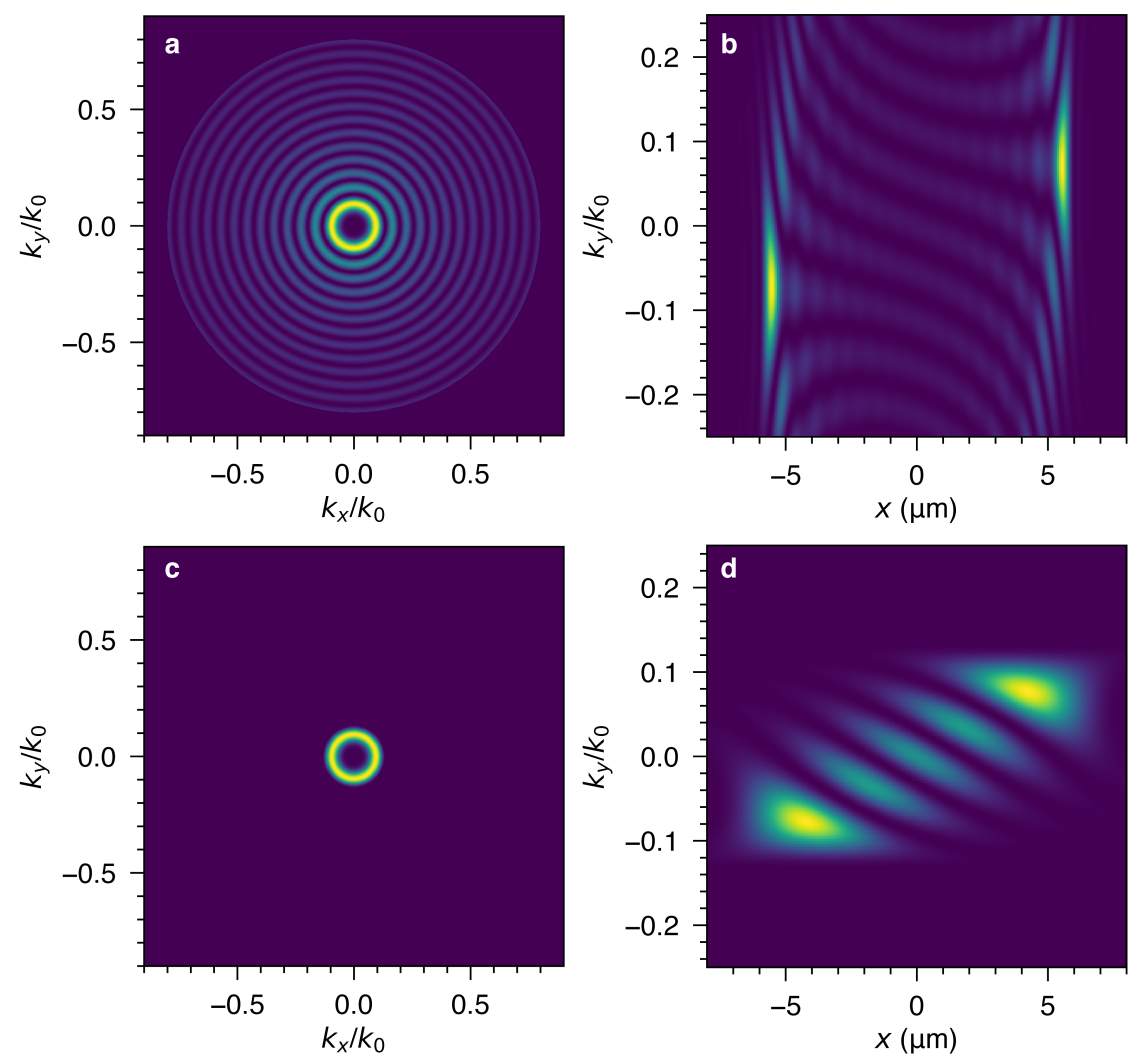

Figure S7. Effect of aperture A2 (see Figure S1) on the resulting cylindrical-lens images. (a) Fourier image of a ring laser with with $r_{\text {ring }}=5.7 \mu \mathrm{m}, \lambda=640 \mathrm{~nm}$, and $p-q-1=4$, calculated by taking the square modulus of eq S10 and multiplying by eq S8. (b) Calculated image formed after focusing (a) with a cylindrical lens. (c) Same as in (a) but clipped by aperture A2 in the Fourier plane. (d) Calculated image formed after focusing (c) with the cylindrical lens.

resembles the patterns observed in Figures 3, 4, and 5 in the main text. The four intensity minima can be clearly observed, and counting them is straightforward. Furthermore, in contrast to the pattern shown in Figure S7b, the intensity in the top left and bottom right corner is minimized, which leads to reduced crosstalk between $l=4$ and $l=-4$ in the measurement. The aperture in the Fourier plane can thus combat detrimental effects of the finite size of the source and allow measurements that are comparable to ones performed on free-space OAM beams, ${ }^{\mathrm{S} 8}$ which do not show phase jumps in the radial direction.

\section{Section S4. Rate-Equation Modelling of Lasing Dynamics}

To confirm and explain our observations from the single-pulse measurements, we modelled the intensity emitted from the different lasing modes with a system of coupled rate equations that are solved for the exciton population in the gain medium and the electric field in the different modes of the ring cavity. Our model is similar to those previously used to describe the physics of ring lasers. ${ }^{\text {S9 }}$ However, to model our multi-mode cavity, we 


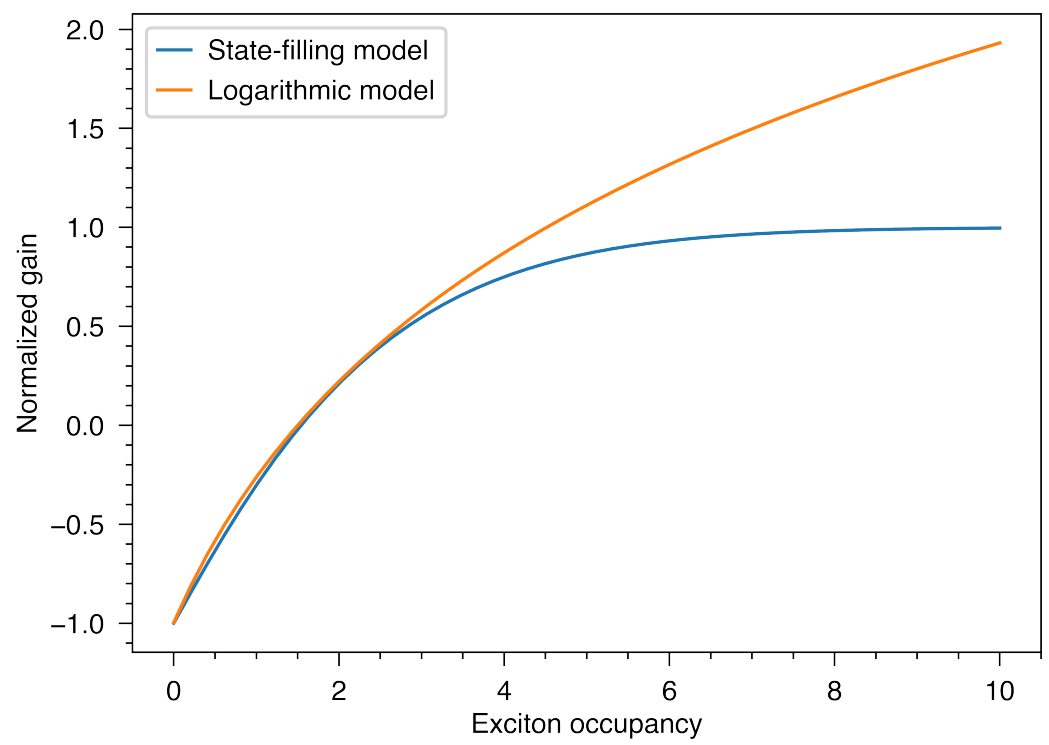

Figure S8. Comparison between a state-filling and a logarithmic gain model.

considered the interaction between more than two modes. We chose two pairs of clockwise and counter-clockwise modes with different frequencies. Additionally, rather than treating the quantum dots (QDs) as a thresholdless four-level gain medium, we used a logarithmic gain function commonly used for semiconductors that are not confined in all three dimensions. ${ }^{\mathrm{S} 10}$ The more accurate expression would be based on state-filling models, ${ }^{\mathrm{S} 11}$ but the logarithmic gain model is in good agreement in the excitation range of interest and has the advantage that it is a simple analytical expression:

$$
g\left(N, g_{0}, N_{\mathrm{s}}, N_{\mathrm{tr}}\right)=g_{0} \log \left(\frac{N+N_{\mathrm{s}}}{N_{\mathrm{tr}}+N_{\mathrm{s}}}\right)
$$

The parameters in our implementation are the average number of excitons per quantum dot, $N$, the normalized gain coefficient, $g_{0}$, and the shift and threshold populations, $N_{\mathrm{s}}$ and $N_{\mathrm{tr}}$. For colloidal quantum dots, gain occurs via state filling and the logarithmic function does not accurately describe the physics. Fortunately, for moderately strong excitation and average exciton occupancies up to $N=4$, eq S18 can match the values predicted by a more complicated state-filling model. We used this comparison to estimate the parameters $N_{\mathrm{s}}, N_{\mathrm{tr}}$, and $g_{0}$ and achieved good agreement for $N_{\mathrm{s}}=1.5, N_{\mathrm{tr}}=1.5$, and $g_{0}=11.5 / 8$, as shown in Figure S8.

In our rate-equation model, the average exciton occupancy is given by:

$$
\dot{N}=\gamma\left[-N-N_{\mathrm{QD}} g\left(N, g_{0}, N_{s}, N_{t r}\right)\left(\left|E_{1}\right|^{2}+\left|E_{2}\right|^{2}+p_{\text {weak }}\left|E_{3}\right|^{2}+p_{\text {weak }}\left|E_{4}\right|^{2}\right)\right] .
$$

Electric fields evolve according to: 


$$
\begin{aligned}
& \dot{E}_{1 / 2}=\left[N_{\mathrm{QD}} g\left(N, g_{0}, N_{s}, N_{t r}\right)-1\right] E_{1 / 2} \pm k_{\mathrm{ud}} E_{2 / 1} \\
& \dot{E}_{3 / 4}=p_{\text {weak }}\left[N_{\mathrm{QD}} g\left(N, g_{0}, N_{s}, N_{t r}\right)-1\right] E_{3 / 4} \pm k_{\mathrm{ud}} E_{4 / 3},
\end{aligned}
$$

where $N$ gives the exciton occupancy per quantum dot. $\gamma$ is the ratio of the photon lifetime to the carrier lifetime, which we estimated to be $10^{-4}$ (the exciton lifetime of our quantum dots is tens of ns while we estimate the photon lifetime in the cavity to be a few ns based on the observed linewidth of $0.5 \mathrm{~nm}$ ). $N_{\mathrm{QD}}$ is the normalized density of quantum dots. $E_{j}$ is the electric field of the $j^{\text {th }}$ mode. Modes 1 and 3 are counter-clockwise-propagating, and modes 2 and 4 are clockwise-propagating. Modes 1 and 2 have the resonance at a different frequency than modes 3 and 4, which causes modes 3 and 4 to have a lowered spectral overlap with the gain medium, modelled by $p_{\text {weak. }}$ The threshold condition for lasing thus becomes $N_{\mathrm{QD}} g\left(N, g_{0}, N_{s}, N_{t r}\right)>1$. The condition for the intrinsic gain threshold of the quantum dots is given by $g\left(N, g_{0}, N_{\mathrm{s}}, N_{\mathrm{tr}}\right)=0$, and happens at $N \approx 1.5$. We experimentally observed the lasing threshold of our devices to be by a factor 1.5 higher than the intrinsic gain threshold of the quantum dots. This we can use, together with the expression for the lasing threshold $\left[N_{\mathrm{QD}} g(1.5 \cdot 1.5,11.5 / 8,1.5,1.5)>1\right]$, to extract $N_{Q D} \approx 3.5$. This is the normalized quantum-dot density necessary to reach lasing at the given threshold. We assumed that our excitation rate is significantly faster than all other rates in the system and accordingly, rather than explicitly writing out a time-dependent excitation rate, we treated it as instantaneous and worked with initial values for the exciton population. As we conducted our experiments $30-50 \%$ above threshold, we chose $N=4$ as the initial value for the exciton occupation. Finally $k_{\text {ud }}$ is a parameter that describes directional coupling caused by the S-shaped coupler. ${ }^{\mathrm{S} 12}$

Our model is not capable of incorporating the stochastic nature of spontaneous emission, which seeds the build-up of the lasing oscillation. To mimic this, we used random initial values for the electric fields in the modes. For $E_{1}$ and $E_{2}$, we sampled from a normal distribution with mean 0.01 and standard deviation 0.0035 . For $E_{3}$ and $E_{4}$, we drew random values from the same distribution but scaled down by a factor 0.85 , to again take into account the lowered overlap with the gain medium. The respective phases were drawn from a uniform distribution. All parameters are summarized in Table S1.

For each geometrical configuration (absence or presence of S-shaped coupler), we numerically solved the time evolution of our system of rate equations 5000 times with random initial values for the electric fields. The integrated emitted electric fields in the different modes can finally be treated and histogrammed in the same way 
as our experimental data. To model our hypothesized effect of the blazed grating, we scaled the output intensities for the clockwise modes by a factor $1 / 3$ for this configuration. Results are compared to experimental results in the main text.

\begin{tabular}{l|l|l|l} 
Parameter & Meaning & Value & Origin of value \\
\hline$N_{\mathrm{tr}}$ & Parameter for gain equation & 1.5 & Comparison with state-filling model \\
\hline$N_{\mathrm{s}}$ & Parameter for gain equation & 1.5 & Comparison with state-filling model \\
\hline$g_{0}$ & Parameter for gain equation & $11.5 / 8$ & Comparison with state-filling model \\
\hline$N_{\mathrm{QD}}$ & $\begin{array}{l}\text { Normalized quantum-dot } \\
\text { density }\end{array}$ & 3.5 & $\begin{array}{l}\text { Matching threshold of device to } \\
\text { quantum-dot gain threshold }\end{array}$ \\
\hline$N$ & Average excitons per dot & dependent & $\begin{array}{l}\text { Dependent variable, } \\
\text { Initial value }=4\end{array}$ \\
\hline$p_{\text {weak }}$ & $\begin{array}{l}\text { Reduced overlap of weaker } \\
\text { mode pair with QD gain }\end{array}$ & 0.98 & $\begin{array}{l}\text { Parameter to match experimental } \\
\text { results }\end{array}$ \\
\hline$E_{1 / 2}$ & $\begin{array}{l}\text { Electric field in strong mode } \\
\text { pair }\end{array}$ & dependent & $\begin{array}{l}\text { Normal distributed initial values, } \\
\mu=0.01, \sigma=0.0035\end{array}$ \\
\hline$E_{3 / 4}$ & $\begin{array}{l}\text { Electric field in weak mode } \\
\text { pair }\end{array}$ & $\begin{array}{l}\text { dependent } \\
\text { Parameter for directional } \\
\text { coupling for blazed grating, }\end{array}$ & $\begin{array}{l}\text { Normal distributed initial values, } \\
\mu=0.0085, \sigma=0.003\end{array}$ \\
\hline$k_{\text {ud }}$ & $\begin{array}{l}\text { Parameter to match experimental } \\
\text { results }\end{array}$ \\
\hline
\end{tabular}

Table S1. Parameters used in our rate-equation model

\section{Section S5. Supplementary References}

(S1) Goodman, J. W. Introduction to Fourier Optics; Roberts and Company Publishers, 2005.

(S2) Novotny, L.; Hecht, B. Principles of Nano-Optics; Cambridge University Press, 2012.

(S3) Baddour, N. Operational and Convolution Properties of Two-Dimensional Fourier Transforms in Polar Coordinates. JOSA A 2009, 26, 1767-1777.

(S4) Zhu, J.; Cai, X.; Chen, Y.; Yu, S. Theoretical Model for Angular Grating-Based Integrated Optical Vortex Beam Emitters. Opt. Lett. 2013, 38, 1343.

(S5) Cai, X.; Wang, J.; Strain, M. J.; Johnson-Morris, B.; Zhu, J.; Sorel, M.; O’Brien, J. L.; Thompson, M. G.; Yu, S. Integrated Compact Optical Vortex Beam Emitters. Science 2012, 338, 363-366.

(S6) Petersen, J.; Volz, J.; Rauschenbeutel, A. Chiral Nanophotonic Waveguide Interface Based on Spin-Orbit Interaction of Light. Science 2014, 346, 67-71.

(S7) Mechelen, T. V.; Jacob, Z. Universal Spin-Momentum Locking of Evanescent Waves. Optica 2016, 3, 118-126.

(S8) Alperin, S. N.; Niederriter, R. D.; Gopinath, J. T.; Siemens, M. E. Quantitative Measurement of the Orbital Angular Momentum of Light with a Single, Stationary Lens. Opt. Lett. 2016, 41, 5019-5022.

(S9) Sorel, M.; Laybourn, P. J. R.; Scirè, A.; Balle, S.; Giuliani, G.; Miglierina, R.; Donati, S. Alternate Oscillations in Semiconductor Ring Lasers. Opt. Lett. 2002, 27, 1992-1994.

(S10) Saxena, D.; Mokkapati, S.; Parkinson, P.; Jiang, N.; Gao, Q.; Tan, H. H.; Jagadish, C. Optically Pumped Room-Temperature GaAs Nanowire Lasers. Nat. Photonics 2013, 7, 963-968.

(S11) Geuchies, J. J.; Brynjarsson, B.; Grimaldi, G.; Gudjonsdottir, S.; van der Stam, W.; Evers, W. H.; Houtepen, A. J. Quantitative Electrochemical Control over Optical Gain in Quantum-Dot Solids. ACS Nano 2020, 15, 377-386.

(S12) Hayenga, W. E.; Ren, J.; Parto, M.; Wu, F.; Hokmabadi, M. P.; Wolff, C.; El-Ganainy, R.; Mortensen, N. A.; Christodoulides, D. N.; Khajavikhan, M. Direct Generation of Tunable Orbital Angular Momentum Beams in Microring Lasers with Broadband Exceptional Points. ACS Photonics 2019, 6, 1895-1901. 